\title{
Effect of Porous Chitosan Microspheres Loaded with Platelet-Rich Plasma and Bone Marrow-Derived Mesenchymal Stem Cells on Regeneration of Tibia Defect
}

\author{
Qi Chen $\mathbb{D D}^{1}{ }^{1}$ Ziru Zhao, ${ }^{2}$ and Guoyong Yin $\mathbb{D}^{3}$ \\ ${ }^{1}$ Department of Orthopedic, The Second Affiliated Hospital of Nanjing Medical University, Nanjing, 210011 Jiangsu, China \\ ${ }^{2}$ Department of Orthopedic, Anting Hospital, JiaDing District, Shanghai, China \\ ${ }^{3}$ Department of Orthopedic, The First Affiliated Hospital of Nanjing Medical University, Nanjing, 210000 Jiangsu, China
}

Correspondence should be addressed to Guoyong Yin; guoyongyin888@sina.com

Received 16 March 2019; Accepted 13 May 2019; Published 13 June 2019

Guest Editor: Jianxun Ding Copyright ( 2019 Qi Chen et al. This is an open access article distributed under the Creative Commons Attribution License, which
permits unrestricted use, distribution, and reproduction in any medium, provided the original work is properly cited.

\begin{abstract}
Objective. Repair of bone defects represents a grave clinical challenge because of the tremendous difficulties in the recovery of bone function and regeneration of bone loss. Therefore, we investigated the effects of platelet-rich plasma-loaded (PRP) porous chitosan microspheres (PCMs) on the differentiation of bone marrow-derived mesenchymal stem cells (BMSCs) and the proliferation and differentiation potential of BMSCs loaded by PCMs in vitro. We also established the model of bone defect repair in rat tibia to further explore the effects of PCMs loaded with PRP and BMSCs on bone regeneration. Methods. MTT assay was used to detect the proliferative ability of BMSCs after hypoxia/reoxygenation (H/R) treatment and the proliferative ability of BMSCs loaded by PCMs; polymerase chain reaction (PCR) was used to detect the expression of alkaline phosphatase (ALP), type I collagen (Col I), and type II collagen (Col II) in BMSCs after hypoxia and in BMSCs induced by PRP-loaded PCMs; PCR was used to detect the expression of Runt-associated transcription factor 2 (Runx2) and osteocalcin (OC) in the newly generated bone tissue; micro-CT scanning was applied to measure the bone mineral density and bone volume of the newly generated bone tissue in rats. Results. BMSCs still have the normal potential of proliferation and differentiation after H/R treatment. PCMs can provide a larger surface for the attachment of BMSCs, facilitating cell proliferation. Loaded by PCMs, PRP can be slowly released, effectively stimulating the differentiation of BMSCs. PCM/PRP/BMSC composites increased the expression levels of Runx2 and OC in the newly generated bone in rat tibia defect and the bone mineral density. Moreover, the composites improved the rate of regenerated bone volume. Conclusion. The application of PCM/PRP/BMSC composites is promising in the repair of tibia defects.
\end{abstract}

\section{Introduction}

Biomaterials such as hydrogels, including porous chitosan microsphere (PCM), and fiber sheets have drawn great attention as tissue engineering scaffolds in bone defect repair thanks to their good biocompatibility and biodegradability [1-3]. Of all biomaterials, chitosan microspheres are used as a typical biomedical material mainly in cell culture, drug carrier, and gene therapy. With a porous structure, PCMs can support cell adhesion and proliferation by incorporating platelet-rich plasma (PRP), which is rich in growth factors. Therefore, PCM has obvious advantages as a tissue engineering scaffold. PCMs prolong the half-life of growth factors and maintain their activity and stability [4]. PCMs can not only load plasma but can also function as a cell carrier by increasing the diameter of the microspheres to induce cell aggregation [5]. By efficiently delivering growth factors and nutrients, PCMs facilitate the migration and adhesion of cells toward osteoblasts and also the proliferation and differentiation of cells into osteoblasts. With these remarkable properties, PCM has the clinical value of applying as a scaffold for bone tissue engineering [6]. PRP is similar to the normal blood in terms of the proportions of growth factors. Containing a variety of growth factors related to bone repair, PRP helps promote bone defect repair, such as growth/differentiation factor 5 (GDF-5), bone morphogenetic protein 2 (BMP- 
2 ), and transforming growth factor $\beta 1$ (TGF- $\beta 1$ ). Increased levels of TGF- $\beta 1$ and BMP- 2 can improve osteogenesis [7, 8]. Bone marrow-derived mesenchymal stem cells (BMSCs) are multipotential stem cells that can differentiate into such cells as osteoblasts and chondrocytes. Therefore, BMSCbased therapies may improve the repair of bone defects [9, 10].

In this study, we explored the effects of PCM-PRP and PCM-BMSC composites on osteogenesis in vivo, demonstrating the cocktail effect of growth factors and BMSCs on bone formation in vivo and in vitro.

\section{Materials and Methods}

2.1. Preparation of Major Reagents and Cell Purchase. Chitosan-gelatin microspheres were prepared based on the method used by Song et al. [11]. Chitosan/ $\beta$-glycerol phosphate solution (solution A) was prepared with chitosan solution $(2.2 \%(v / v)$ acetic acid solution) and $\beta$-glycerol phosphate solution $(50 \%(w / w)$ aqueous solution) in a $v / v$ ratio of $5: 1$. Type I collagen solution (solution B) was prepared by adding $700 \mu \mathrm{l}$ of type I collagen into the mixture of $30 \mu \mathrm{l}$ of $0.1 \mathrm{~mol} / \mathrm{l}$ sodium hydroxide solution and $100 \mathrm{ml}$ of 10x D-Hanks. Solution A and solution B were mixed in a $v / v$ ratio of $1: 1$ after filter sterilization. The mixed solution was gelled at $37^{\circ} \mathrm{C}$ for 15 minutes, froze in a $-80^{\circ} \mathrm{C}$ refrigerator for 2 hours, and freeze-dried for 48 hours before $2.5 \%$ glutaraldehyde was added to crosslink proteins for 3 hours at room temperature. After that, PBS was used to wash the mixture twice and diluted ethanol was added to dehydrate the mixture to obtain chitosan-gelatin microspheres. PCM-PRP composites were prepared based on the method used by Deprés-Tremblay et al. [12]. The mice were decapitated to obtain blood samples, which were anticoagulated with $12.9 \mathrm{mM}$ sodium citrate. The blood samples were then centrifuged at $160 \mathrm{~g}$ for 10 minutes using ACE E-Z PRPTM centrifuge. The supernatants and 1-2 mm of red blood cell pellets were taken and centrifuged at $400 \mathrm{~g}$ for $10 \mathrm{~min}$ at room temperature before $0.5 \mathrm{ml}$ of materials at the bottom of the tube were resuspended to prepare PRP. PCMs were then mixed with PRP at a $v / v$ ratio of $1: 1$ to obtain PCM-PRP composites. To prepare PCM-BMSC composites, we purchased a MC3T3-E1 cell line (ATCC, USA) as BMSCs. PCMs were incubated with $5 \times 10^{6}$ cells $/ \mathrm{ml}$ of BMSC solution for 3 days to collect PCM/BMSC composites.

2.2. Animal Grouping and Modeling. A total of 20 male Wistar rats aged 4-8 weeks were divided into the Sham group, Model group, PRP/BMSC group, and PCM/PRP/BMSC group. The Sham group received sham surgery on the left tibia. The Model group received surgery to create a $3 \mathrm{~mm}$ diameter hole on the left tibia. The PRP/BMSC group received surgery to create a $3 \mathrm{~mm}$ diameter hole on the left tibia, and then, PRP and BMSCs were placed into the hole. For the PCM/PRP/BMSC group, PCM-PRP and PCMBMSC composites $(1: 2, v / v)$ were implanted in the defect after a $3 \mathrm{~mm}$ diameter hole was made on the left tibia. Each of these implants contained approximately $10^{4}$ BMSCs and $0.1 \mathrm{ml}$ PRP. After 2 months and 4 months of treatment, respectively, 5 rats were euthanized in each group, and the newly generated bone tissues at the site of bone defect were collected.

2.3. Expression of Cytokines Related to Osteogenesis. Alkaline phosphatase (ALP), type I collagen (Col I), and type II collagen (Col II) are key marker proteins during the differentiation of BMSCs into osteoblasts. Runt-associated transcription factor 2 (Runx2) and osteocalcin (OC) are key osteogenic genes for bone regeneration in bone defects [13]. Cells or tissue specimens were collected to extract RNA according to the instructions of the EASYspin Plus Bone Tissue RNA Kit. The mRNA expression levels of osteogenesisrelated cytokines, including ALP, Col I, and Col II, and genes, including Runx 2 and $\mathrm{OC}$, were detected by quantitative realtime RT-PCR (qRT-PCR) using GAPDH as internal reference.

2.4. Measurement of Bone Mineral Density and Bone Volume. Tibiae were separated and scanned using micro-CT scanning. The bone mineral density and bone volume of newly generated bones were calculated.

2.5. Cell Proliferation. According to the MTT assay, $2^{\times} 10^{3}$ cells were seeded in a 96-well plate. MTT solution was prepared by adding MTT (Sigma; EMD Millipore) into phosphate-buffered saline. A total of $20 \mu \mathrm{g} / \mathrm{l}$ MTT solution was added to each well, and the cells were cultured for another $4 \mathrm{~h}, 8 \mathrm{~h}$, and $16 \mathrm{~h}$, respectively, before their viability was measured by the absorbance value $(A)$ using a microplate reader at a wavelength of $490 \mathrm{~nm}$.

2.6. Statistical Analysis. All data were processed using SPSS 20.0 software package. Measurement data were expressed as the mean \pm standard deviation and compared using a $t$-test. A $P$ value of $<0.05$ was considered to be statistically significant.

\section{Results}

3.1. Proliferation and Differentiation Potential of BMSCs after Hypoxia/Reaeration Treatment. To simulate the environment of chitosan microspheres prior to the sustained release of BMSCs, these stem cells were subjected to hypoxia/reoxygenation (H/R) treatment. The proliferative ability of BMSCs after H/R treatment was detected using the MTT. The results showed that there was no significant difference in MTT absorbance between H/R-treated cells and the control cells $(P>0.05)$, as shown in Figure 1. Studies have shown that the use of PRP can enhance the expression of osteogenicrelated genes. After BMSCs were subjected to H/R treatment, PRP was used to induce cell differentiation for 7 days before the collection of total RNA. The intracellular expression levels of ALP, Col I, and osteopontin (OPN) were measured by qRT-PCR to evaluate the potential of cells differentiating into osteoblasts. The results showed that there were no significant differences in the expression levels of ALP, Col I, and OPN between H/R-treated BMSCs and PRP-induced BMSCs (all $P>0.05$ ), as shown in Figure 2. This suggests that the 

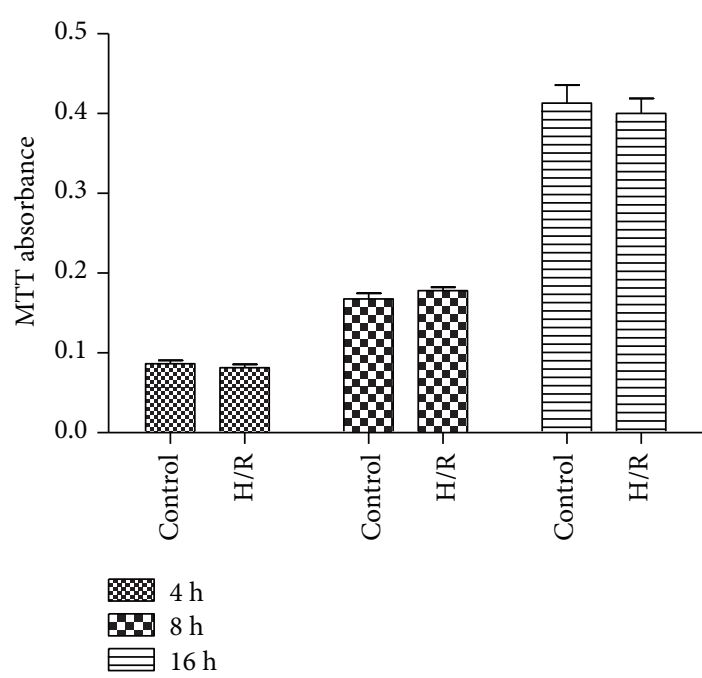

FIgURE 1: Cell viability of BMSCs after H/R treatment.

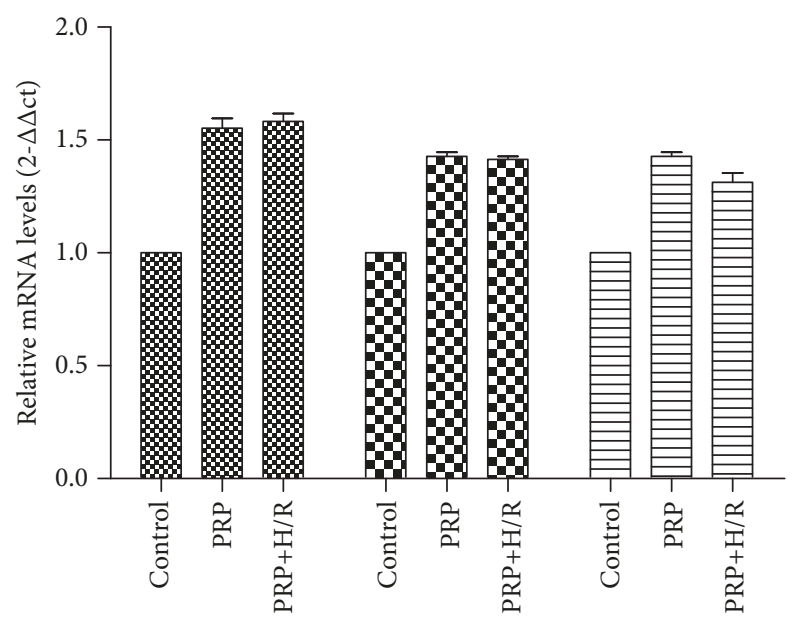

\%

E. Col I

Col II

Figure 2: Expression of ALP, Col I, and Col II in BMSCs after H/R treatment.

differentiation potential of BMSCs does not reduce when BMSCs are loaded by PCMs.

3.2. Cell Growth of PCM-BMSC Composites. BMSCs were cultured with PCMs for 3 days to prepare PCM/BMSC composites, and spherical particles were observed under the microscope. After the PCM/BMSC composites were cultured for another 7 days, only a fraction of irregular PCM residues was observed under the microscope. MTT assay was performed for PCM/BMSC composites. The results showed that the cell viability of BMSCs loaded by PCMs was higher $(P<0.05)$, and the results of MTT assay were shown in Figure 3. This suggests that before the degradation of PCMs, they can provide a larger surface for the attachment of BMSCs, facilitating cell proliferation.

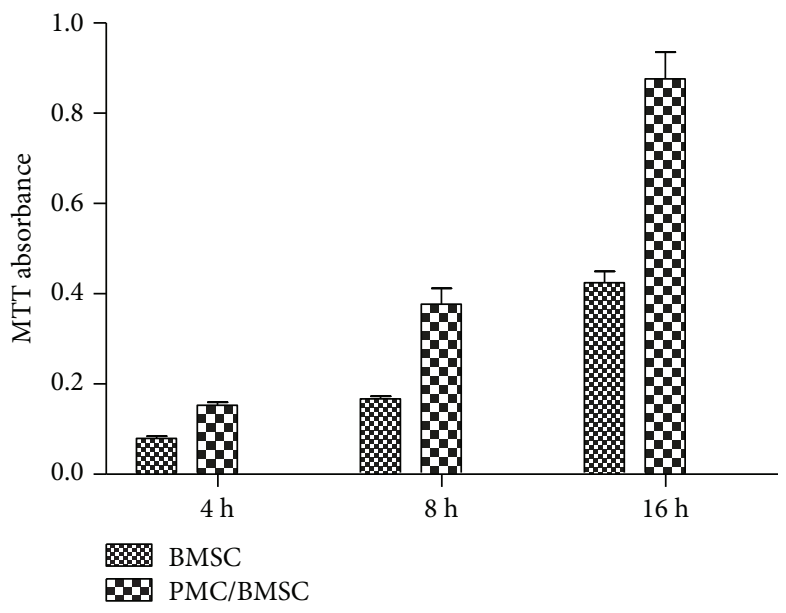

Figure 3: PCMs promote growth of BMSCs.

3.3. Effect of PCM/PRP Composites on Differentiation of BMSCs In Vitro. We first prepared PCM/PRP composites by incorporating PRP into PCMs. Total RNA was collected after in vitro induction of differentiation of BMSCs by PCM/PRP composites for 7 days. The intracellular expression levels of ALP, Col I, and OPN were detected by qRT-PCR. The results showed that the expression levels of ALP, Col I, and OPN in BMSCs induced by PCM/PRP composites were significantly increased compared with PRP-induced BMSCs $(P<0.05)$, as shown in Figure 4 . This suggests that PCMs allow the sustained release of PRP, which is better in promoting the differentiation of BMSCs.

3.4. PCM/PRP/BMSC Composites Increased Bone Mineral Density in Rat Tibia Defect. We constructed a rat tibia defect model and used micro-CT scanning to detect the bone mineral density of the tibia defect. According to the results, the $\mathrm{PRP} / \mathrm{BMSC}$ and PCM/PRP/BMSC groups had a significant increase in bone mineral density at 2 and 4 months after model establishment compared with the Model group $(P<0.05)$; the bone mineral density of the PCM/PRP/BMSC group was higher than that of the PRP/BMSC group $(P<0.05)$ and was closed to that of the Sham group $(P>0.05)$, as shown in Figure 5.

3.5. PCM/PRP/BMSC Composites Increased the Rate of Regenerated Bone Volume in Rat Tibia Defect. We used micro-CT scanning to detect the cortical bone thickness of the tibia defect, and the rate of regenerated bone volume is equal to the thickness of the regenerated cortical bone over the thickness of the cortical bone of the Model group. According to the results, the PRP/BMSC and $\mathrm{PCM} / \mathrm{PRP} / \mathrm{BMSC}$ groups had a significant increase in bone volume at 2 and 4 months after model establishment compared with the Model group $(P<0.05)$; the bone volume of the PCM/PRP/BMSC group was higher than that of the PRP/BMSC group $(P<0.05)$ and reached about $92.6 \%$ of that of the Sham group $(P>0.05)$, as shown in Figure 6. 


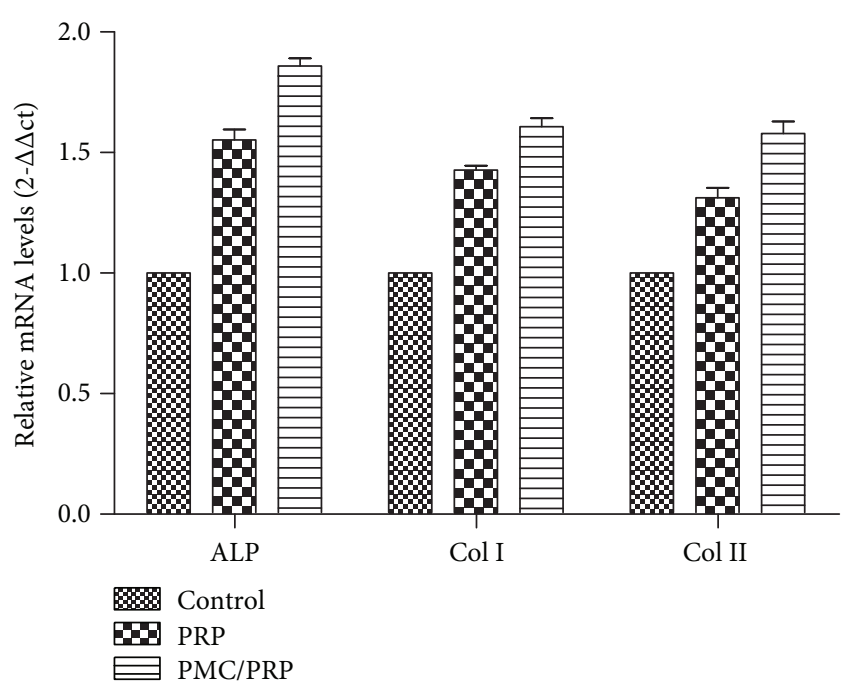

Figure 4: Expression of ALP, Col I, and Col II in BMSCs induced by $\mathrm{PCM} / \mathrm{PRP}$ composites in vitro.

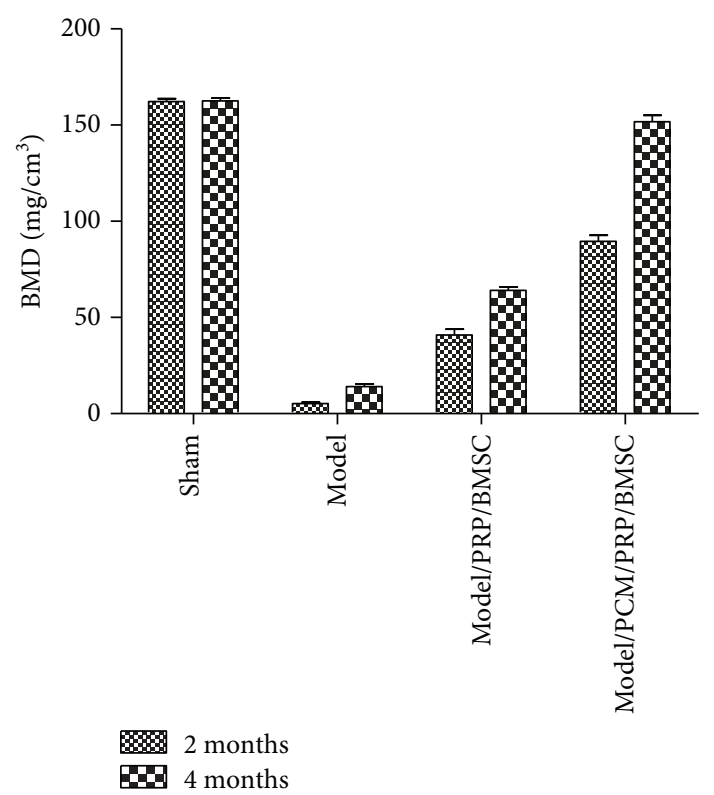

FIGURE 5: PCM/PRP/BMSC composites increase bone mineral density in rat tibia defect.

3.6. PCM/PRP/BMSC Composites Increased the Expression of Runx2 and OC in Rat Tibia Defect. The regenerated tissue at the defect was collected 2 months after modeling to measure the expression of Runx 2 and OC by PCR. The results showed that Runx2 and OC were expressed in the Model, PCM/PRP/BMSC, and PRP/BMSC groups $(P<0.05)$. The expression levels of Runx 2 and OC in the PCM/PRP/BMSC group were higher than those of the PRP/BMSC group $(P<0.05)$, as shown in Figure 7.

\section{Discussion}

Biomaterials have received wide attention because of the properties like slowing drug release in tissues, inducing

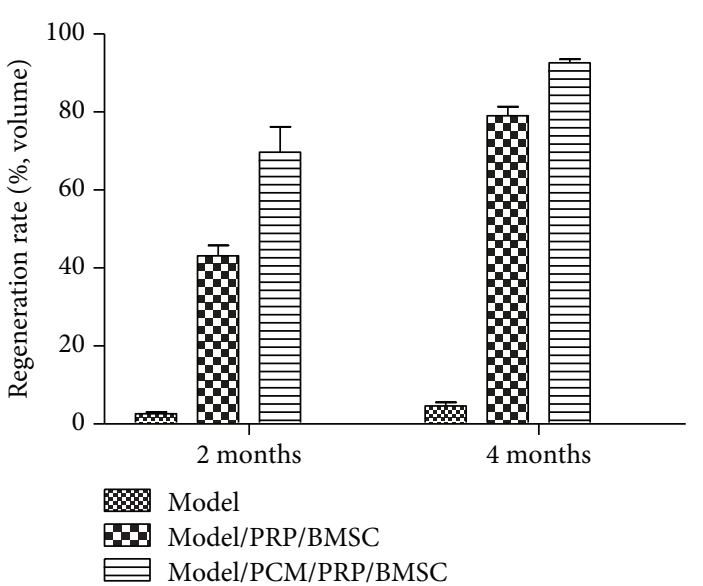

FIGURE 6: PCM/PRP/BMSC composites increase the rate of regenerated bone volume in rat tibia defect.

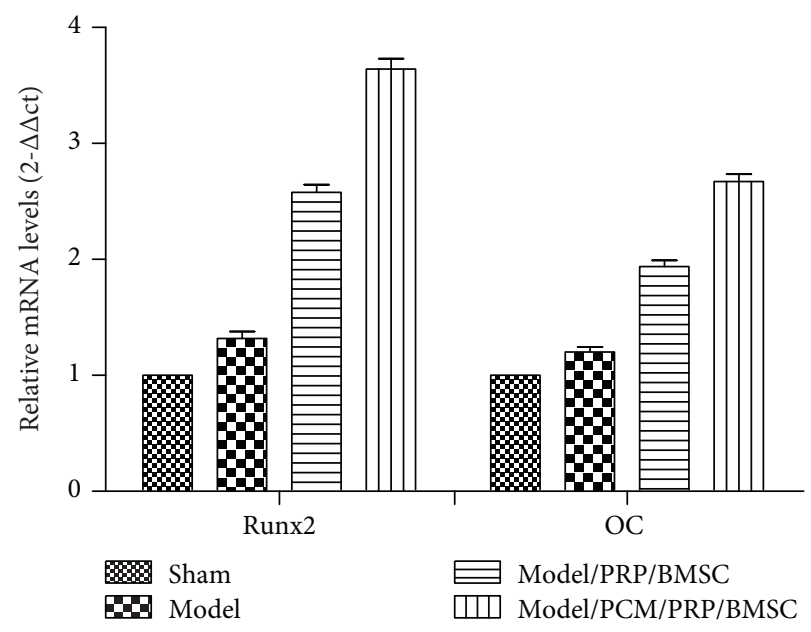

Figure 7: Expression of Runx2 and OC in rat tibia defect.

inflammatory responses, and recruiting immune cells or the capability of acting as scaffolds [14, 15]. Clinically, regeneration of bone defect is an intractable problem. The development of bone tissue engineering has a great significance for the regeneration of bone defects. However, due to the low bioactivity and poor hydrophilicity, the scaffold is not an ideal choice for the regeneration of bone defects. Therefore, biomaterials such as chitosan and gelatin are applied to bone defect regeneration and have received extensive attention [16]. PCM is a natural nontoxic biomaterial with good biocompatibility and biodegradability. This study used a combination of PCM-PRP and PCM-BMSC composites for the repair of rat tibia defects to evaluate their bone repair effects. We first proved that BMSCs in PCM-BMSC composites still have the ability of proliferation and differentiation and PCMPRP composites are capable of inducing the differentiation of BMSCs. While BMSCs are capable of self-renewal and differentiation into osteoblasts, PRP is prominent in promoting osteogenesis, allowing it to induce the proliferation and differentiation of BMSCs into osteoblasts. Most of the endogenous cytokines peak at the early stage of bone fracture repair and are metabolized thereafter; moreover, part of the 
endogenous cytokines, such as BMP-2, has to reach a certain concentration before they can promote osteogenesis [17]. After bone injury, exogenous cytokines can be supplied in an amount more sufficient than endogenous cytokines, but the direct use of exogenous cytokines is of limited effect due to the inactivation by enzymes in body fluids [18]. In addition, high dose of exogenous cytokines may also be counterproductive for it would promote osteoclastogenesis, thereby accelerating bone resorption and resulting in reduced bone mass at the injury site [19]. Therefore, an appropriate release of exogenous cytokines is an important factor for the induction of effective bone regeneration [20]. In this study, PCMs were used to load PRP and BMSC, respectively, which slowed down the release of PRP and BMSCs and improved the biological effect of PRP. In this study, we combined the advantages of BMSCs and PRP in bone regeneration after injury to optimize the treatment for bone defects in rat tibiae. Such a combination has produced satisfying results in tissue regeneration in some studies. Lian et al. found that the combination of BMSCs and PRP contributes to the repair and regeneration of diabetic wounds [21].

In conclusion, the use of PMC/PRP/BMSC composites benefits the repair of rat tibia defect. Its application is promising in the repair of tibia defects.

\section{Data Availability}

All the data are available in the handwritten notebook documented in our lab or available from the corresponding author upon request.

\section{Conflicts of Interest}

The authors declare no conflicts of interest.

\section{Acknowledgments}

This work was supported by Shanghai Jiading District Science and Technology Commission Project (JDKW2018-W03).

\section{References}

[1] Y. Cheng, C. He, J. Ding, C. Xiao, X. Zhuang, and X. Chen, "Thermosensitive hydrogels based on polypeptides for localized and sustained delivery of anticancer drugs," Biomaterials, vol. 34, no. 38, pp. 10338-10347, 2013.

[2] J. Wu, K. Zhang, X. Yu, J. Ding, L. Cui, and J. Yin, "Hydration of hydrogels regulates vascularization in vivo," Biomaterials Science, vol. 5, no. 11, pp. 2251-2267, 2017.

[3] J. Zhang, T. Zheng, E. Alarçin et al., "Porous electrospun fibers with self-sealing functionality: an enabling strategy for trapping biomacromolecules," Small, vol. 13, no. 47, article 1701949, p. 13, 2017.

[4] S. Yu, X. Xu, J. Feng, M. Liu, and K. Hu, "Chitosan and chitosan coating nanoparticles for the treatment of brain disease," International Journal of Pharmaceutics, vol. 560, no. 14, pp. 282-293, 2019.

[5] R.-X. Shao, R.-F. Quan, T. Wang et al., "Effects of a bone graft substitute consisting of porous gradient $\mathrm{HA} / \mathrm{ZrO}_{2}$ and gela- tin/chitosan slow-release hydrogel containing BMP-2 and BMSCs on lumbar vertebral defect repair in rhesus monkey," Journal of Tissue Engineering and Regenerative Medicine, vol. 12, no. 3, pp. e1813-e1825, 2018.

[6] A. Ali and S. Ahmed, "A review on chitosan and its nanocomposites in drug delivery," International Journal of Biological Macromolecules, vol. 109, no. 1, pp. 273-286, 2018.

[7] D. Yang, S. Moon, and D.-W. Lee, "Surface modification of titanium with BMP-2/GDF-5 by a heparin linker and its efficacy as a dental implant," International Journal of Molecular Sciences, vol. 18, no. 1, p. 229, 2017.

[8] S. Kasagi and W. Chen, "TGF-beta1 on osteoimmunology and the bone component cells," Cell \& Bioscience, vol. 3, no. 1, p. 4, 2013.

[9] D. Zou, Z. Zhang, D. Ye et al., "Repair of Critical-Sized Rat Calvarial Defects Using Genetically Engineered Bone Marrow-Derived Mesenchymal Stem Cells Overexpressing Hypoxia-Inducible Factor-1 $\alpha$," Stem Cells, vol. 29, no. 9, pp. 1380-1390, 2011.

[10] Y. Zhang, F. Wang, J. Chen, Z. Ning, and L. Yang, "Bone marrow-derived mesenchymal stem cells versus bone marrow nucleated cells in the treatment of chondral defects," International Orthopaedics, vol. 36, no. 5, pp. 1079-1086, 2012.

[11] K. Song, L. Li, X. Yan et al., "Characterization of human adipose tissue-derived stem cells in vitro culture and in vivo differentiation in a temperature-sensitive chitosan/ $\beta$ glycerophosphate/collagen hybrid hydrogel," Materials Science and Engineering, vol. 70, Part 1, pp. 231-240, 2017.

[12] G. Deprés-Tremblay, A. Chevrier, N. Tran-Khanh, M. Nelea, and M. D. Buschmann, "Chitosan inhibits platelet-mediated clot retraction, increases platelet-derived growth factor release, and increases residence time and bioactivity of platelet-rich plasma in vivo," Biomedical Materials, vol. 13, no. 1, article 15005, p. 1, 2018.

[13] W. Q. Xu, J. X. Ding, C. X. Xiao, L. Y. Li, X. L. Zhuang, and X. S. Chen, "Versatile preparation of intracellular-acidity-sensitive oxime-linked polysaccharide-doxorubicin conjugate for malignancy therapeutic," Biomaterials, vol. 54, pp. 72-86, 2015.

[14] Y. Wang, Z. Jiang, W. Xu et al., "Chiral Polypeptide thermogels induce controlled inflammatory response as potential immunoadjuvants," ACS Applied Materials \& Interfaces, vol. 11, no. 9, pp. 8725-8730, 2019.

[15] B. Zhu, W. Xu, J. Liu, J. Ding, and X. Chen, "Osteoinductive agents-incorporated three-dimensional biphasic polymer scaffold for synergistic bone regeneration," ACS Applied Materials \& Interfaces, vol. 5, no. 2, pp. 986-995, 2018.

[16] J. H. Ye, Y. J. Xu, J. Gao et al., "Critical-size calvarial bone defects healing in a mouse model with silk scaffolds and SATB2-modified iPSCs," Biomaterials, vol. 32, no. 22, pp. 5065-5076, 2011.

[17] S. Pattnaik, S. Nethala, A. Tripathi, S. Saravanan, A. Moorthi, and N. Selvamurugan, "Chitosan scaffolds containing silicon dioxide and zirconia nano particles for bone tissue engineering," International Journal of Biological Macromolecules, vol. 49, no. 5, pp. 1167-1172, 2011.

[18] D. Lindhorst, F. Tavassol, C. von See et al., "Effects of VEGF loading on scaffold-confined vascularization," Journal of Biomedical Materials Research Part A, vol. 95, no. 3, pp. 783-792, 2010.

[19] F. Pati, B. Adhikari, and S. Dhara, "Collagen intermingled chitosan-tripolyphosphate nano/micro fibrous scaffolds for 
tissue-engineering application," Journal of Biomaterials Science. Polymer Edition, vol. 23, no. 15, p. 16, 2012.

[20] B.-B. Seo, J.-T. Koh, and S.-C. Song, "Tuning physical properties and BMP-2 release rates of injectable hydrogel systems for an optimal bone regeneration effect," Biomaterials, vol. 122, pp. 91-104, 2017.

[21] Z. Lian, X. Yin, H. Li et al., "Synergistic effect of bone marrowderived mesenchymal stem cells and platelet-rich plasma in streptozotocin-induced diabetic rats," Annals of Dermatology, vol. 26, no. 1, pp. 1-10, 2014. 


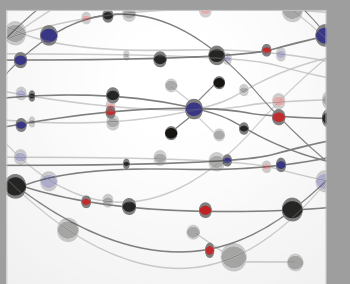

The Scientific World Journal
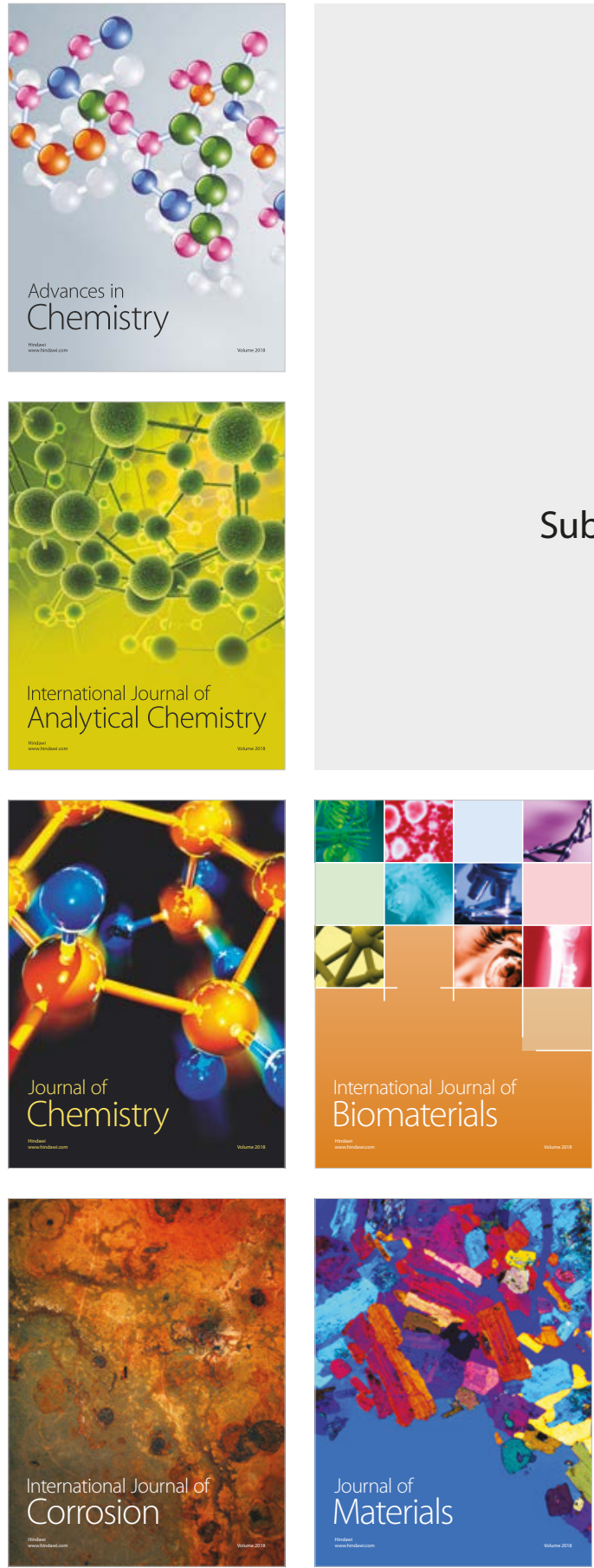

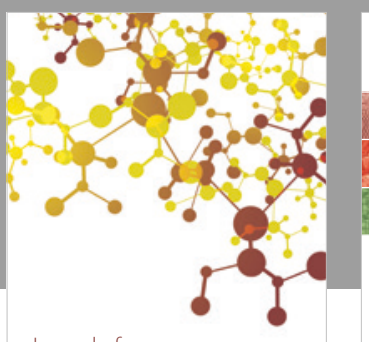

Journal of

Applied Chemistry
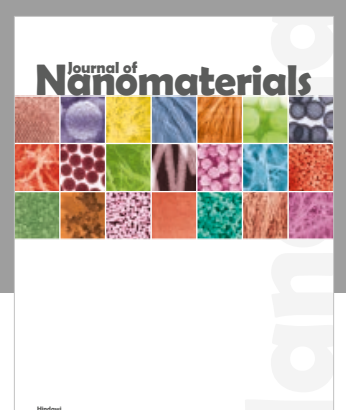

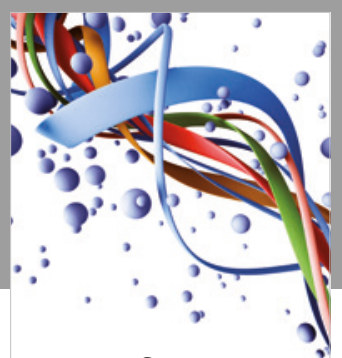

Scientifica

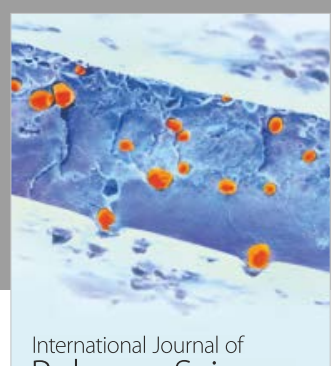

Polymer Science

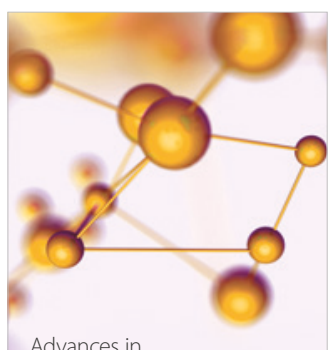

Physical Chemistry
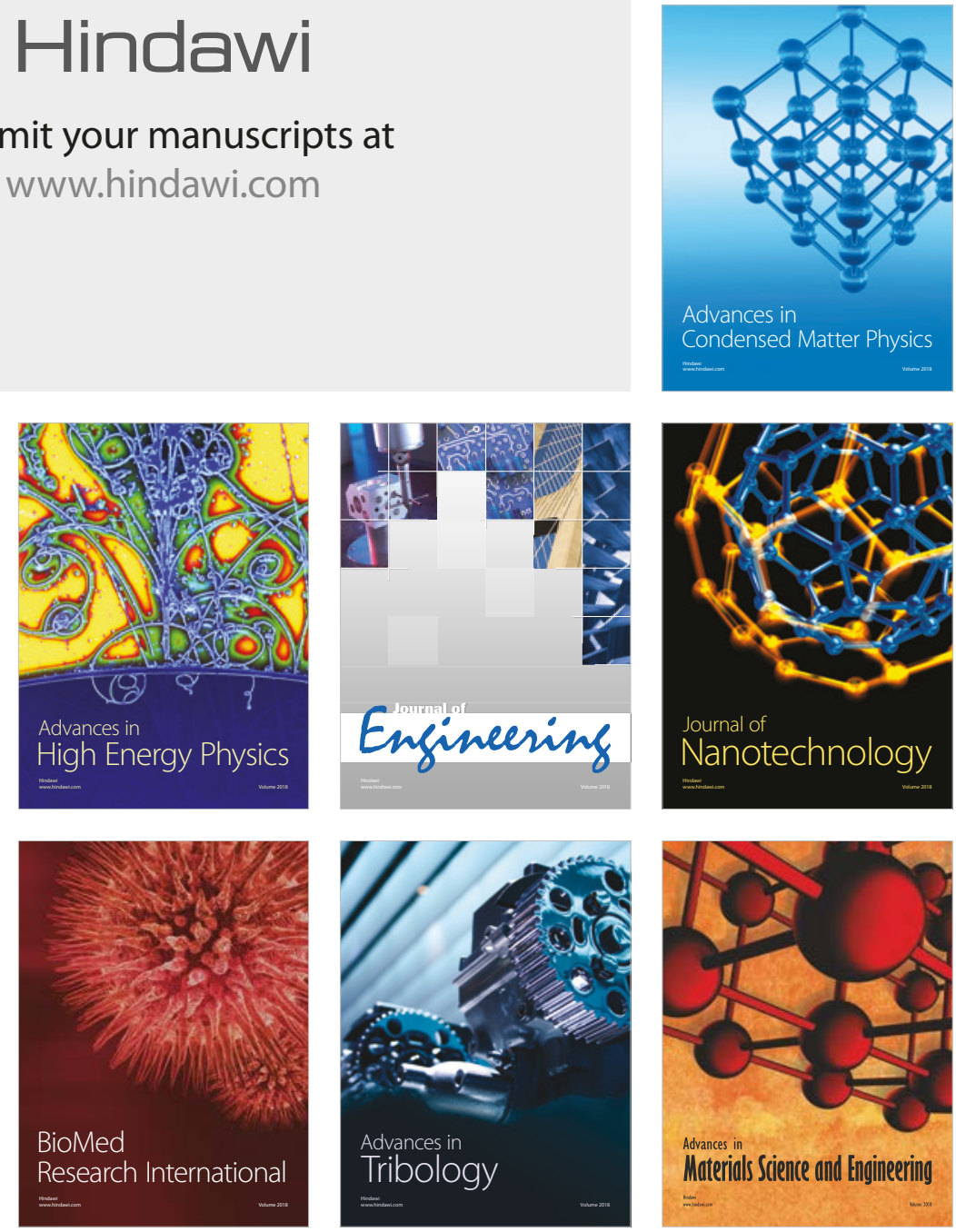